\title{
Enhancing efficiency of using water due to explosive breakup of liquid drop
}

\author{
Anastasia G. Borisova ${ }^{1}$, Vladislav M. Gumerov ${ }^{1}$, Maxim V. Piskunov ${ }^{1, *}$, and Denis V. \\ Ushmaev $^{1}$ \\ ${ }^{1}$ National Research Tomsk Polytechnic University, 634050 Tomsk, Russia
}

\begin{abstract}
Using high-speed video recording, the experiments were performed to research quantitative characteristics of explosive breakup phenomenon of $5-15 \mu 1$ water droplets containing $2 \times 2 \times 1 \mathrm{~mm}$ and $2 \times 2 \times 2$ $\mathrm{mm}$ solid inclusions, when heated in a tube furnace at temperatures of 1070-1370 K. Experimental results report number and size of the droplets detached during explosive breakup. We show that the fragmentation of liquid layer covering solid particles facilitates the increase the evaporation surface area 15 -fold versus the initial surface area of a drop.
\end{abstract}

\section{Introduction}

Experimental studies [1,2] report the phenomenon of explosive breakup of liquid layers of heterogeneous water drops (liquid volume $-5-15 \mathrm{mcl}$, size of single solid inclusion is 50 $70 \%$ of liquid volume), which is implemented by the intensive evaporation at the interface of solid particle / liquid at high temperatures. Fig. 1 illustrates processes of heating and explosive breakup of heterogeneous drop. Analysis of research results $[1,2]$ allows us to select conditions facilitating the process of explosive breakup. To provide practical applications discussed in $[1,2]$ with data on quantitative characteristics of the explosive breakup, it is necessary to estimate such parameters in experiments as number, size and total surface area of the liquid after breakup. Such information will contribute to the enhancing resource efficiency, energy efficiency of high-temperature thermal treatment technologies of liquids [3] and firefighting by liquids with impurities [4].

The aim of this paper is to estimate number, size and surface area increase in the process of explosive breakup of heterogeneous liquid drops at high-temperature heating.

\footnotetext{
*Corresponding author: piskunovmv@tpu.ru
} 


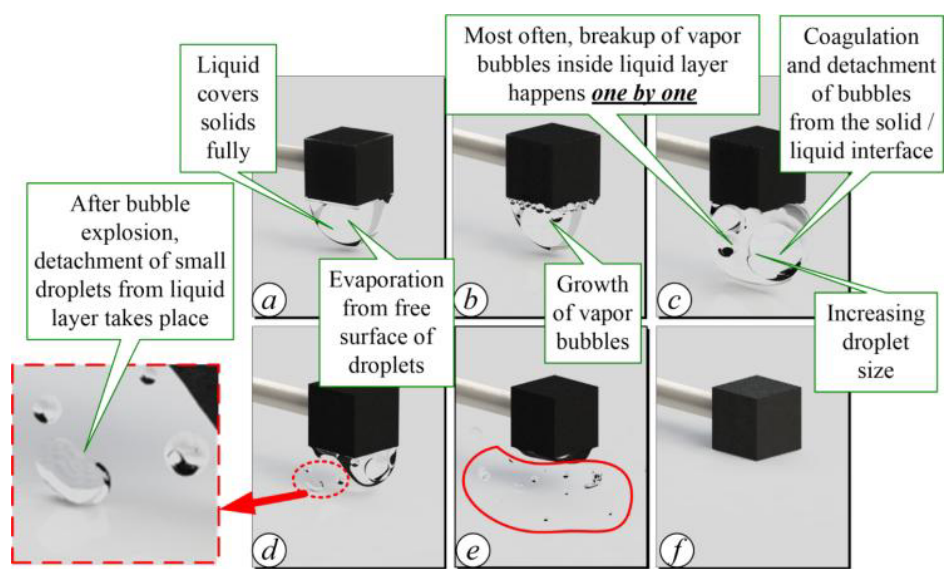

Fig. 1. Scheme of explosive breakup of heterogeneous liquid drop.

\section{Experimental setup and research methods}

Experimental procedure is generally similar to that used in $[1,2]$. The main difference is a using the tube furnace to produce the high-temperature area. The tube furnace has an integral regulator and type $S$ thermocouple, which provide the temperature monitoring and the demanded temperature regime. Temperature varies in the range of 1073-1373 K. Importantly, the tube furnace is equipped with the heating tube. Such design enables to install high-speed camera from the one side and to introduce a heterogeneous drop in heating area from the other one. Heating of a drop is performed at the center of the tube.

The heterogeneous drop consists of the solid particle made of natural graphite in shapes of cube and parallelepiped with dimensions $2 \times 2 \times 1 \mathrm{~mm}$ and $2 \times 2 \times 2 \mathrm{~mm}$, as well as the water layer (water volume $-5-15 \mathrm{mcl}$; temperature $-298 \mathrm{~K}$ ), which covers an inclusion fully. As in $[1,2]$ the solid particle is mechanically fixed on the thin rod made of ceramics. Then, the dosing device produces drops of the required volumes. Introducing heterogeneous liquid drop - thin rod system into the high temperature air in the tube body is implemented by the automatic mechanism. High-speed video recording allows determining linear dimensions of, for example, the formed small water droplets. The recorded video data are processed by Phantom Camera Control software. Together with drop size, owing to high recording speed the number of the droplets formed during the breakup is determined visually. These obtained data are used to calculate the total area $S_{\text {out }}$ of the formed water droplets. This parameter basically characterizes increase of evaporation surface area. Errors of measuring dimensions of the droplets formed during the breakup are not more than 0.001. A series of the experiments at identical conditions includes seven tests, which are performed in the mentioned temperature range with step of $50 \mathrm{~K}$. In each series, size of solid particles and initial water volume vary.

\section{Results and discussion}

Figs. 2 and 3 illustrate the influence of the ratio between initial volumes of water and inclusion for different dimensions of the latter on the increase of the total evaporation surface area due to the breakup of the liquid layer. Note that the maximum number of small droplets corresponds to the minimum ratio between initial volumes of water and inclusion. This effect is the most obvious for the droplets with the size up to $0.5 \mathrm{~mm}$ (Fig. 3). 


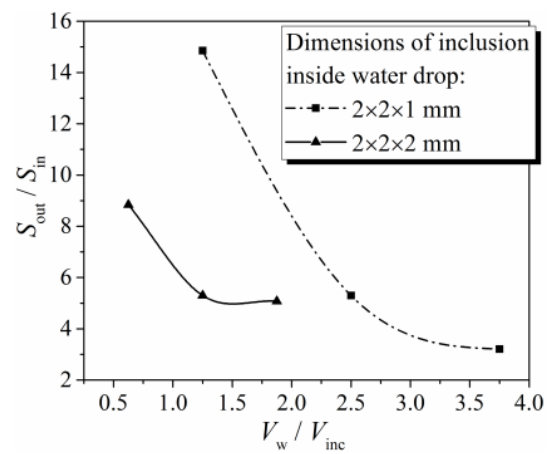

Fig. 2. $S_{\text {out }} / S_{\text {in }}$ versus $V_{\mathrm{w}} / V_{\text {inc }}$ for three initial volumes of water $(5 \mu 1,10 \mu 1$ and $15 \mu 1)$ and two dimensions of inclusions $(2 \times 2 \times 1 \mathrm{~mm}$ and $2 \times 2 \times 2 \mathrm{~mm})$.

Taking into account experimental data in Figs. 2 and 3, the maximum ratio of $S_{\text {out }} / S_{\text {in }}$ will be under condition of $V_{\mathrm{w}} \sim V_{\text {inc }}$ or even $V_{\mathrm{w}}<V_{\text {inc }}$. If the introduction of additional volume of solids is difficult or inappropriate, then the selection of optimum ratio between $V_{\mathrm{w}}$ and $V_{\text {inc }}$ can be implemented by using the data reported in Figs. 2 and 3.

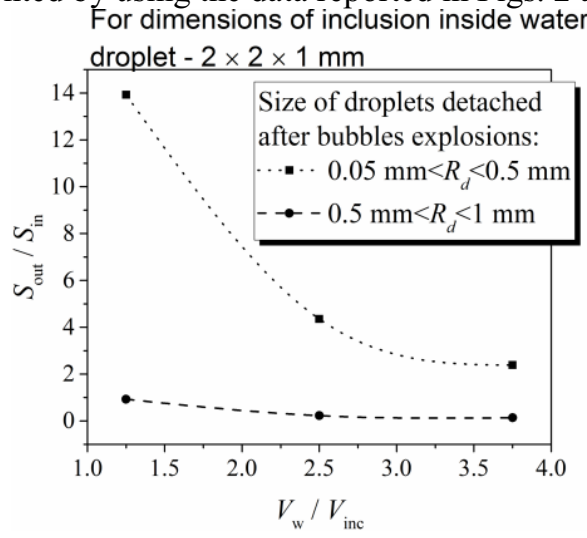

Fig. 3. $S_{\text {out }} / S_{\text {in }}$ versus $V_{\text {w }} / V_{\text {inc }}$ for three initial volumes of water $(5 \mu 1,10 \mu 1$ and $15 \mu 1)$ and inclusion dimensions of $2 \times 2 \times 1 \mathrm{~mm}$ (taking into account the size distribution of the formed droplets)

\section{Conclusion}

After explosive breakup of the liquid layer covering solid particles into small droplets the evaporation surface area increases 15 -fold versus the initial surface area of a drop.

The investigation was supported by the grant (MD-1221.2017.8) and scholarship (SP1049.2016.1) of President of the Russian Federation.

\section{References}

1. G.V. Kuznetsov, M.V. Piskunov, P.A. Strizhak, Int. J. Heat Mass Tran. 92, 360 (2016)

2. R.S. Volkov, G.V. Kuznetsov, M.V. Piskunov, P.A. Strizhak, J. Heat Transf. 138, $014502(2016)$

3. R.S. Volkov, G.V. Kuznetsov, P.A. Strizhak, Int. J. Thermal Sci. 88, 193 (2015)

4. D.I. Kofman, M.M. Vostrikov, A.V. Antonenko, Chem. Pet. Eng. 45, 577 (2009) 